\title{
Evaluation System of Heating Quality
}

\author{
Dongdong $\mathrm{Mu}^{\mathrm{a}}$, Guofeng Wang ${ }^{\mathrm{b}^{*}}$, Yunsheng Fan ${ }^{\mathrm{c}}$ and Cunhe $\mathrm{Li}^{\mathrm{d}}$ \\ IT College of Dalian Maritime University, Dalian, 116026, China \\ amu_dong@yeah.net, ${ }^{\mathrm{b}}$ gfwangsh@163.com , ${ }^{\mathrm{c}}$ fan_yunsheng@163.com, ${ }^{\mathrm{d}}$ licun.he@163.com
}

Keywords: heating quality; web networking and the internet of thing; evaluation system

Abstract: With the increasing levels of automation, building of heating's automatic level have a considerable development. So, quality of heating has been greatly improved. However, in actual operation remain exist extensive management. So, heating field need a heating quality evaluation system to evaluate the quality of heating. The system should monitor the real-time situation and dispatch the energy distribution, then give an evaluation and guidance of heating building [1]. To solve the above problem, this thesis design a system which is basing on web networking and the internet of thing. It can monitor the operation of heating system and accord to the actual heat consumption, expected heat consumption and indoor temperature calculate a score. It has been verified by actual operation: the system can reasonable and effective analyze data, and give a reasonable score of heating quality.

\section{Introduction}

Central heating has a positive effect to energy conservation and improving the urban environment, it is increasingly becoming an important part of the urban public utilities. With the increasing levels of automation in heating industry, to a certain extent, energy-saving and central heating works have achieved automatic control. But in the actual process of building's heating, there are still existing the phenomenons that low level of information and serious waste heat. It can lead to not fully grasp the heating system operating status and energy distribution [2]. There are several reasons lead to this result. Firstly, some managers does not pay enough attention to the heat supply's information. They think that as long as residents don't complain, the heating quality is good. Secondly, lacking of in-depth data analysis, the data is not saving energy, and making full advantage of data can lead to save energy. Finally, there is lacking of a quantitative evaluation system which is a scientific and regulatory information platform. For the above questions, basing on web networking and the internet of things, designing a system which is to evaluate the energy consumption of heat supply.

The system not only can monitor heating system but also base on the least squares algorithm to forecast the consumption of heat. Finally, it can base on the historical data, meteorological index and forecast energy consumption to get a score of heating quality. Examples from the recent literature and a practical application to a university in dalian show that the system can get a reasonable score basing on previous heating data, and the analysis result of the heating has a good guidance effect.

\section{Data Flow}

\section{Data Acquisition and Transmission}

The data acquisition system basing on the internet of thing, it includes perception layer, network layer and application layer [3]. The function of the perception layer is mainly used for collecting information. In this system, it mainly application is to use the PLC and sensor to collect the data from heating system, such as supply water temperature, backwater temperature, flow rate, and the indoor temperature, etc. Main functions of the network layer are depending on the Internet(all kinds of cable network, WiFi, VPN channel), the communication network ( 3 G, GPRS), and other basic network facilities to transfer the information which is from the perception layer. It can transmit the data to the WEB server. The main functions of application layer are that information processing, 
data mining analysis and providing feedback information service. Application service layer mainly include common supporting platform and application system. Common supporting platform include cloud computing platform, high performance computing platform, and physical resource platform. Platform application system include government application system, enterprise application systems. Application service layer is a complete set of system of the terminal, and it is a system of the mind. Through the internet, you can monitor the operation of the system at any time and place. In this thesis, this system is mainly used to store heating data, and using the WEB platform to monitor the status of heating system and data analysis. It can provide real-time information and guidance to managers. The structure of system is shown in Fig. 1.

\section{Web Platform}

The collection of data are stored in real-time database. In order to protect the safety of the realtime database and not all data need to analyze [4]. So, between the real time database and WEB publishing database have a data transmission module. It not only can selectively transmit data but also can prevent hacker's intrusion. It can protect the security of database and internal LAN. Heating quality evaluation system include real time date module, historical data module, and analysis date module. In order to prevent the platform's source code form divulging and protect the security of the platform, the network of outside need to pass a encryption lock to access the platform [5].Platform structure is shown in Fig.2.

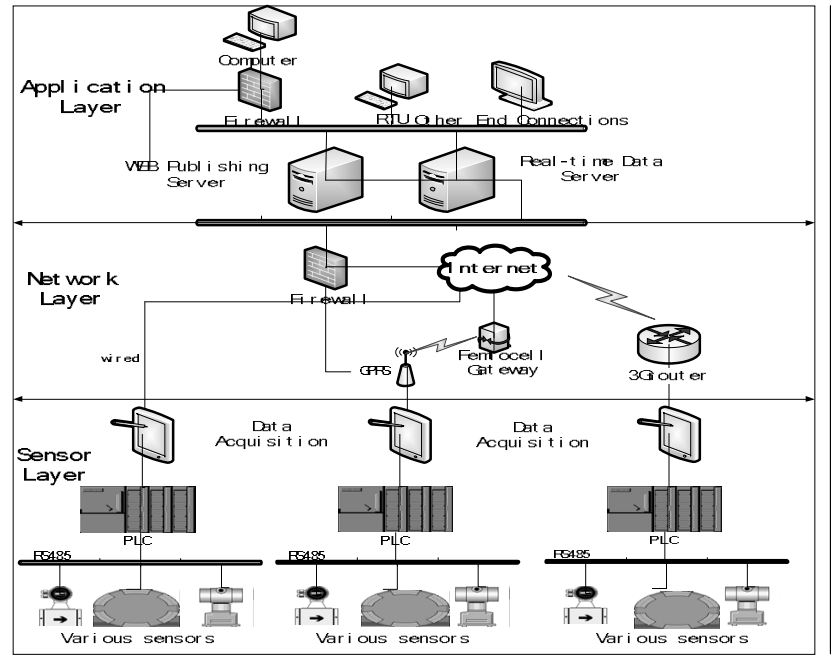

Fig. 1. Structure of system

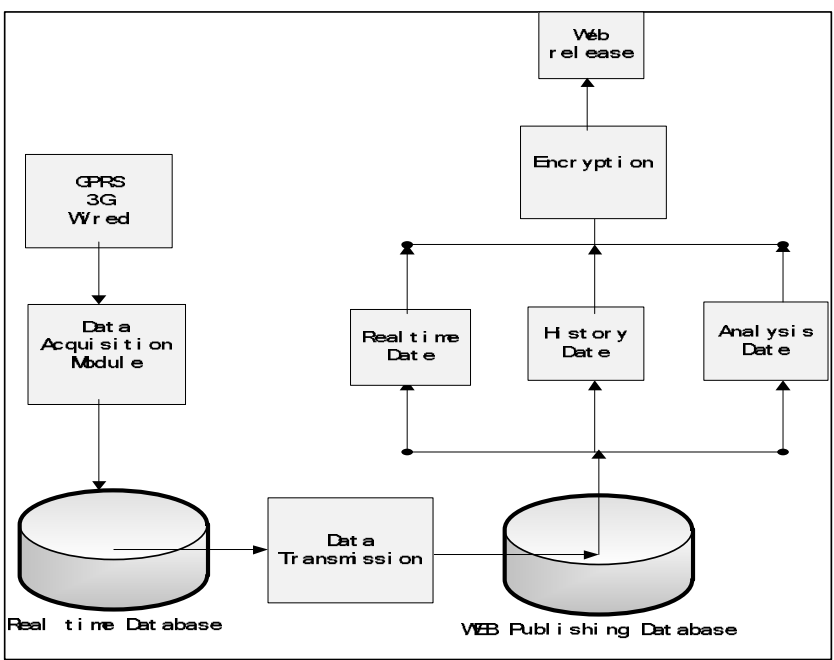

Fig. 2. Platform structure

\section{Set Up Building Heating Evaluation Model}

The system use degree day theory to describe the indoor and outdoor temperature, and use WD(Watt Degree) to describe the heating intensity. According to the experience of heating, heating intensity and WD are direct proportion relationship. So, the WD and HDD(Hot Degree Days) are direct proportion relationship [6]. According to "the urban heating system evaluation standard ", during heating period, indoor room,bedroom and sitting room's temperature should not be less than $18{ }^{\circ} \mathrm{C}$. For simplicity of implementation, standard temperature is set to $18{ }^{\circ} \mathrm{C}$.

HDD is shown in Eq. 1.

$$
H D D=\left(T_{D}-T_{\mathrm{i}}\right)
$$

HDD is degree day, $T_{i}$ is the average daily temperature, $T_{D}$ is reference temperature $18^{\circ} \mathrm{C}$.

WD is shown in Eq. 2. 


$$
W D=\frac{Q_{g}}{\Delta t^{*} S}
$$

WD is watt per square meter. This paper use it to describe the heat intensity. $\mathrm{Q}_{\mathrm{g}}$ is heating load, $\Delta \mathrm{t}$ is the heating time, $\mathrm{S}$ is building area.

A building of a university in dalian, analyze its heating history data and use least square method to analyze WD and HDD. The result of the analysis is $\mathrm{Y}=0.78 \mathrm{X}$. 0.78 is called as coefficient of HDD. $\mathrm{Y}$ is the WD and $\mathrm{X}$ is the WD. According to the result of fitting, calculate the energy of heating consumption which is expected. In the matlab to draw the actual WD and expected WD. The result is show in Fig.3. Because of HDD and WD are directly proportional relationship, building insulation ability is presented by coefficient of HDD. That is to say, different building have different coefficient of HDD, the smaller coefficient, the better insulation ability.

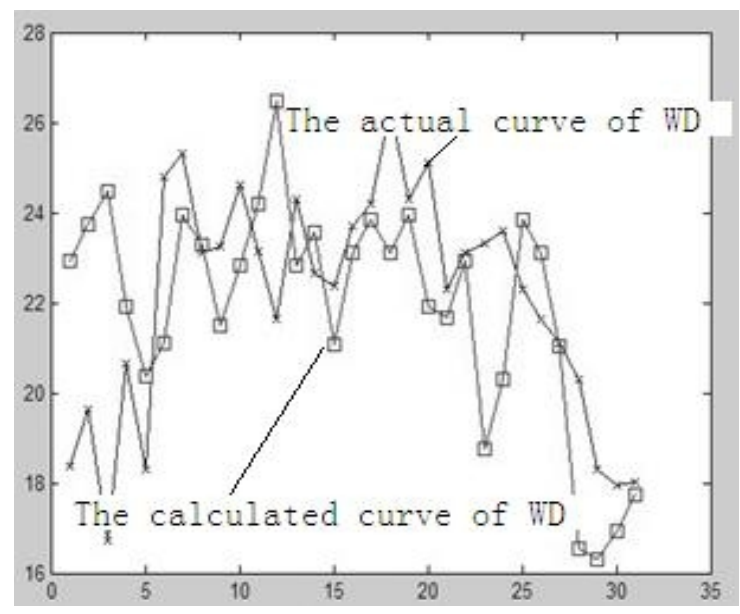

Fig.3. Matlab fitted curve

Using the WD of calculated and the actual HDD, calculate the heat of theory.

$H D D^{*} \mathrm{a}=W_{\mathrm{y}}$

$H D D * \mathrm{a} * S * 24 * 60 * 60=Q_{y}$

a is the coefficient of HDD, $\mathrm{W}_{\mathrm{y}}$ is the WD of calculated, $\mathrm{Q}_{\mathrm{y}}$ is the heat of theory.

The scoring principle of this system is that ignore human factors and only consider the actual data. Score is divided into "whether indoor temperature is up to standard" and "whether energy consumption is up to the standard" two parts. Under the single criterion comparative judgment matrix constructed by expert investigation method, and the indoor temperature's score and energy consumption's score account for $0.6,0.4$ respectively [7].

$$
\begin{gathered}
t_{f}=\left(1-\frac{\left|T_{\mathrm{i}}-18\right|}{18}\right) * 100 \\
\mathrm{n}_{f}=\left(1-\frac{\left|Q_{y}-Q_{g}\right|}{Q_{y}}\right) * 100 \\
\mathrm{z}_{\mathrm{f}}=0.6 * t_{f}+0.4 * n_{f}
\end{gathered}
$$

$\mathrm{t}_{\mathrm{f}}$ is the score of room temperature, $\mathrm{nn}_{\mathrm{f}}$ is the score of energy, $\mathrm{z}_{\mathrm{f}}$ is total score. 


\section{EVALUATION AND GUIDANCE}

Indoor temperature is not only related with heating but also related to many factors [8], such as the switch of door and window and so on. Under the premise of without considering other factors, this platform only according to the actual data which is collected to quantitative analysis heating situation [9].

In a month, the average room temperature curve is shown in Fig. 4. According to room temperature curve and the Eq. 5, indoor temperature score is calculated. Indoor temperature Score is shown in Fig. 5.

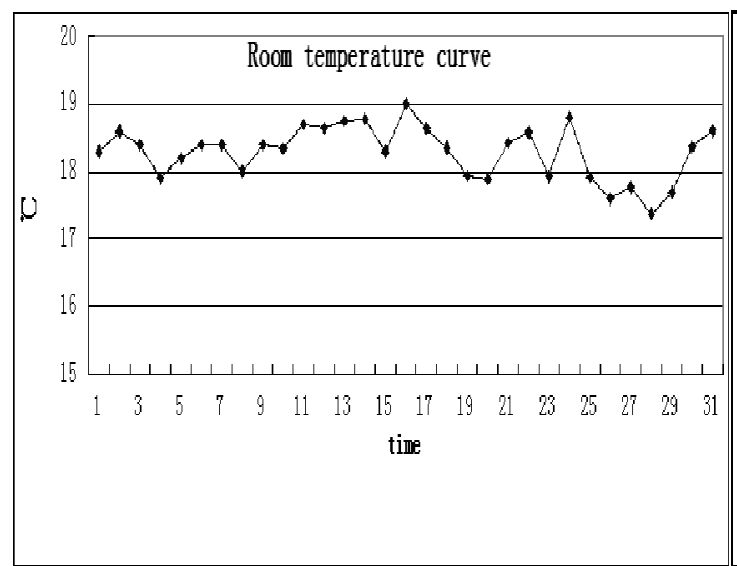

Fig. 4. Indoor Daily Average Temperature

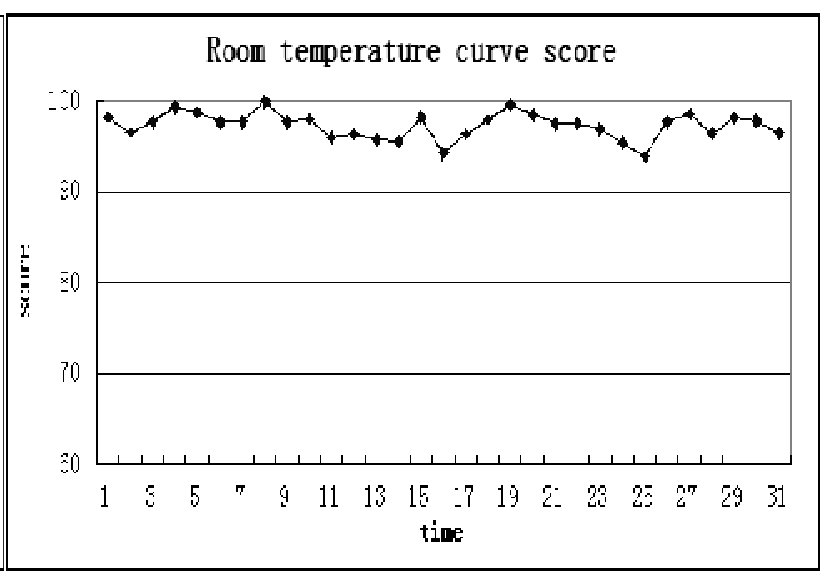

Fig. 5. Indoor Temperature Score

By Fig. 4 \& 5, it can conclusion that the indoor temperature fundamentally stable at $18{ }^{\circ} \mathrm{C}$. It only have small amplitude wave. So the overall score about room temperature is higher, and it is always more than 95 points.

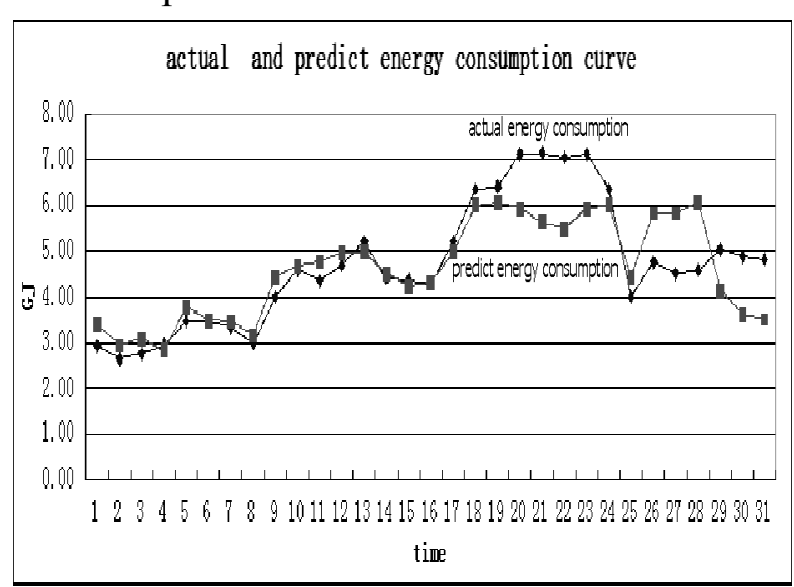

Fig. 6. Actual and Prediction Energy Consumption

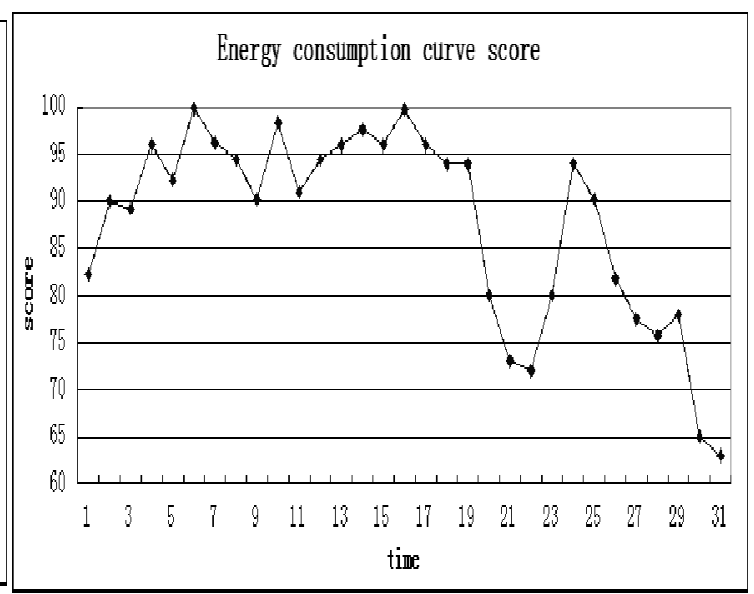

Fig. 7. Energy Consumption Score

According to the Fig. 6, calculate the daily energy consumption's score. The result is shown in Fig. 7.

By Fig. $6 \&$ 7, due to the actual value and the predicted value gradually become consistent, the score of energy consumption is getting higher. But in the latter part of this month, the actual value and the predicted value do not match, so the energy consumption get a lower score. In this month, the average score of room temperature is 96.4, and energy consumption get 87.6. Refer to Eq. 7, calculate the total average score. The result is shown in Fig. 8. 


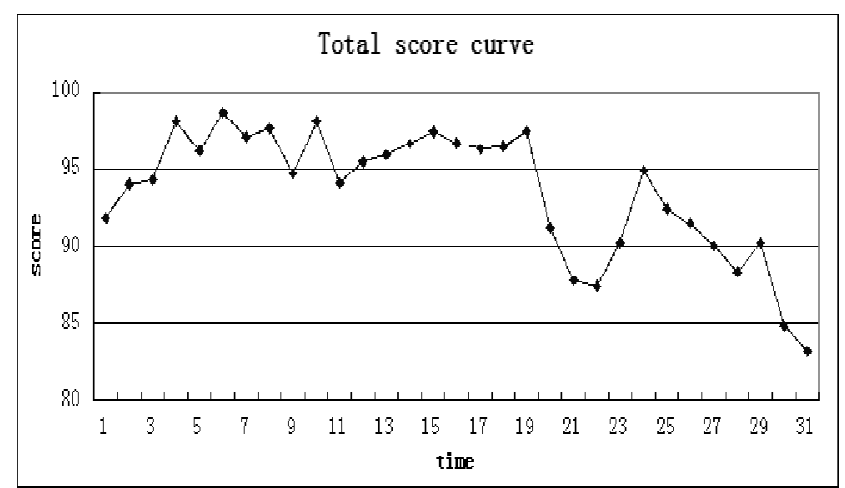

Fig. 8. Total Score

This month, the daily average score is 92.1 . But it not means that the heating quality is very good this month. By Fig. 8 can summarize that the early heating condition is better than later in this month, and need to change the heating of offering in the next month.

\section{CONCLUSION}

The evaluation system of heating quality can fully effective analysis historical data, and establish a reliable evaluation model. Rely on this model can give a reasonable score. According to the weather forecast, managers can provide guidance on future heating basing on the situation before. It can realize the rolling optimization control parameters and help management grasp the overall situation. This system has a good set of industry promotional. It not only can be applied in the field of heating but also chemical industry, traffic and metallurgy, etc.

\section{Acknowledgments}

This work is supported by Natural Science Foundation of Liaoning Province of China under Grant 2015025009, and Fundamental Research Funds for the Central Universities under Grant 3132015039.

\section{References}

[1] Verbeeck G, Hens H. Energy savings in retrofitted dwellings: economically viable?[J]. Energy and buildings, 2005, 37(7): 747-754.

[2] Siyuan Wang, Bingyu Yin, Wenbiao Wang. Heating energy conservation evaluation system and energy saving modeling research[J]. ENERGY CONSERVATION.2013.10:28-32.

[3] Atzori L, Iera A, Morabito G. The internet of things: A survey[J]. Computer networks, 2010, 54(15): 2787-2805.

[4] Qingqi Pei, Yulong Shen, Jianfeng Ma. Summary of the wireless sensor network security technology[J]. COMMUNICATIONS, 2007, 28(8): 113-122.

[5] Chao Li. Research and Design of Networked Control Systems[D]. Hebei:North China electric power university,2012.

[6] Pérez-Lombard L, Ortiz J, Pout C. A review on buildings energy consumption information[J]. Energy and buildings, 2008, 40(3): 394-398.

[7] Shan Zhang. Research of Performance Evaluation System of Heating Boilers[D].Dalian:Dalian maritime university,2013.

[8] Bessa V M T, Prado R T A. Reduction of carbon dioxide emissions by solar water heating systems and passive technologies in social housing[J]. Energy Policy, 2015, 83: 138-150.

[9] Zhongtang $\mathrm{Xu}$, Qingli Liu. Urban heat by heat metering and charging approach is imperative[J]. Urban Development, 2000, 1: 7-10. 